Ann. Biol. anim. Bioch. Biophys., I972, 12 (4), 667-672.

NO'TE

\title{
CONJONCTIF ET CROISSANCE MUSCULAIRE
}

\author{
O. SCHMITT et B.-L. DUMONT \\ avec la collaboration technique de Thérèse DEGAS \\ Laboratoire de Recherches sur la Viande, \\ Centre national de Recherches zootechniques, $I, N, R$. A., \\ 78350 Jouy en Josas
}

Avec l'âge, depuis la naissance, les muscles subissent d'importantes modifications morphologiques qui affectent sensiblement au cours de la croissance leurs dimensions et leur masse.

Ces transformations s'accompagnent de changements dans leur composition et leur texture. A cet égard on note chez les bovins - comme dans d'autres espèces de mammifères - un important accroissement du diamètre moyen des fibres musculaires. Dans cette espèce on enregistre, d'autre part, une diminution de l'importance relative du collagène, plus abondant chez les jeunes que chez les sujets âgés (Wilson et al., 1954; Lawrie, 1961; Goll et al., I963; Vognarová et al., I968), et d'une structure moléculaire différente (Golt et al., I964; KoPP, I97I).

Sur le plan histologique on a montré (Vognarová et al., I968) que bien que les faisceaux de fibres soient de plus faibles dimensions chez les veaux, par rapport aux sujets plus âgés, l'épaisseur du périmysium interfasciculaire y est plus grande. Ces résultats, observés uniquement au niveau des faisceaux primaires ne permettent pas de rendre compte de l'évolution que subit, au sein du muscle, la trame de tissu conjonctif dans son ensemble, et, en particulier, le réseau de conjonctif qui délimite les unités d'organisation du muscle (Schmitt et Dumont, I969).

La présente note rapporte les observations faites lors de la comparaison de la trame du tissu conjonctif du muscle semi-tendinosus d'un veau femelle de race Frisonne pie-noive sacrifiée le jour de sa naissance et d'une femelle bovine de même race âgée de 13 ans. Les deux animaux ont été abattus au C. N. R. Z. Après dissection de la cuisse on a pesé le muscle semi-tendinosus et prélevé dans sa partie médiane une tranche destinée à l'examen histologique selon la technique précédemment exposée (Schmitt et Dumont, I969). Des sections de I5 $\mu$ intéressant la totalité de la section transversale du muscle ont été colorées à la méthode de la pichro-fuschine de Van Gieson. Ces coupes ont été utilisées comme négatifs pour l'obtention d'inter-négatifs puis des documents photographiques qui illustrent ce travail (fig. I à 4).

La figure $\mathrm{I}$ indique en vraie grandeur la surface de section du muscle semi-tendinosus des deux animaux. La figure 2 présente, en réduction, le muscle de l'animal âgé, ramené à la dimension de celui de l'animal à la naissance. Enfin les figures 3 et 4 représentent une vue partielle agrandie de la surface de section des muscles considérés. La coupe de l'animal à la naissance (fig. 3) est ramenée par une projection homothétique à la dimension de la coupe de l'animal âgé.

Il est évident (fig. I) que l'accroissement de masse que subit (entre les deux stades considérés) le muscle semi-tendinosus s'accompagne d'une très large augmentation de la surface de section. La trame générale de conjonctif qui y délimite les unités d'organisation du muscle (ou myoskhènes) paraît, relativement à la surface, plus importante chez le nouveau-né que chez le sujet âgé. Bien que cette trame générale ne corresponde pas à la totalité du tissu conjonctif présent dans le muscle et ne permette qu'une estimation par défaut de l'importance de ce dernier on 


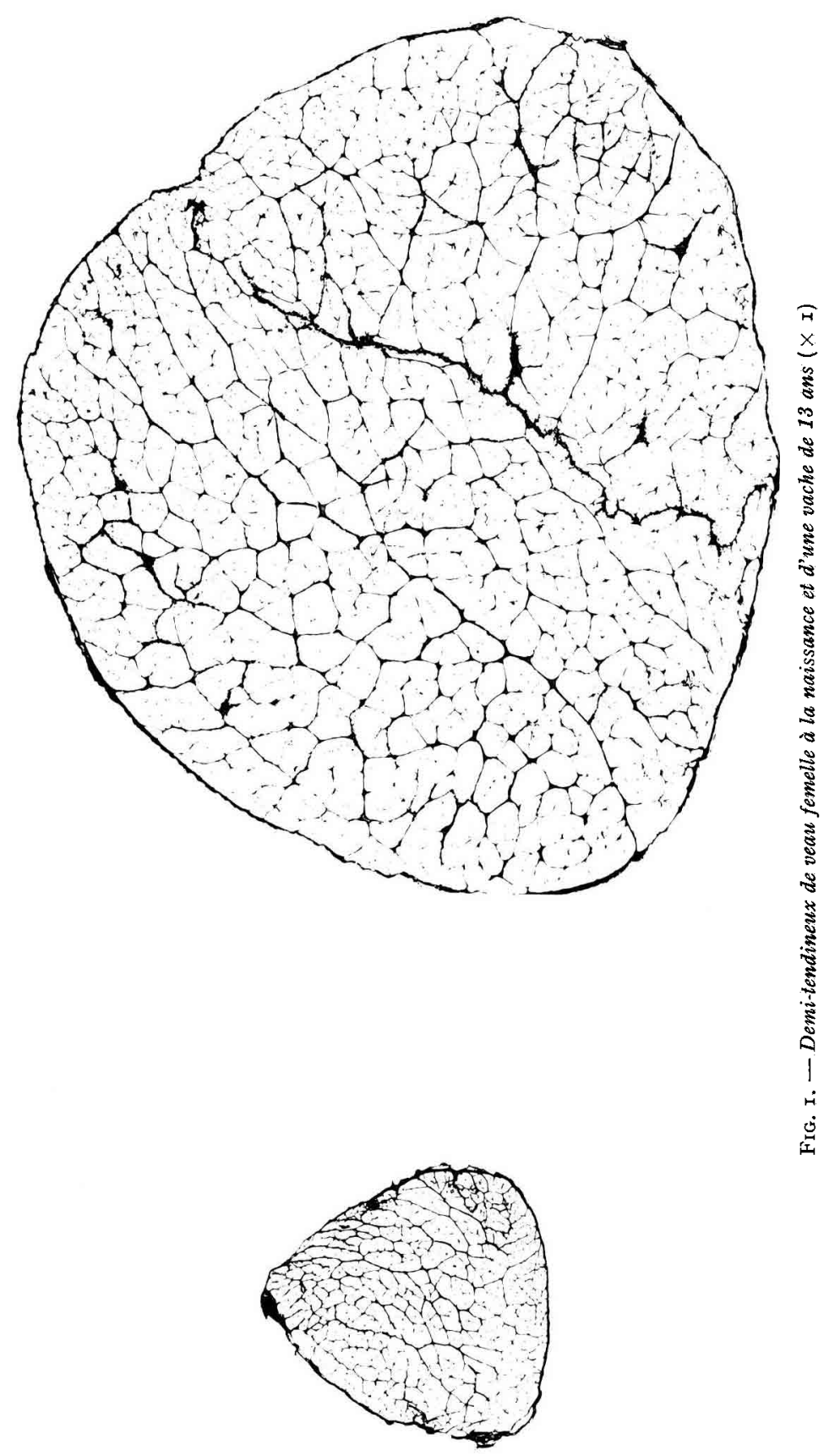


peut toutefois avancer que les observations histologiques, en ce qui concerne la part relative du collagène dans le muscle, vont dans le même sens que les résultats des analyses chimiques rapportées dans la littérature (cf. Supra). On note d'autre part que chez le nouveau-né le réseau du tissu conjonctif paraît plus régulier, plus continu alors que chez le sujet âgé la trame délimite de façon plus tranchée les différentes unités d'organisation.

La figure 2, dans laquelle le muscle de vache est ramené à la taille de celui du veau ne fait pas apparaître de grosses différences entre les deux sujets, sauf une importance légèrement plus forte de la trame conjonctive. Par contre la comparaison des figures 3 et 4 met clairement en évidence les modifications que subit dans sa structure morphologique microscopique la trame générale du tissu conjonctif, ainsi que les modifications qui affectent le périmysium interfasciculaire au niveau des faisceaux primaires.
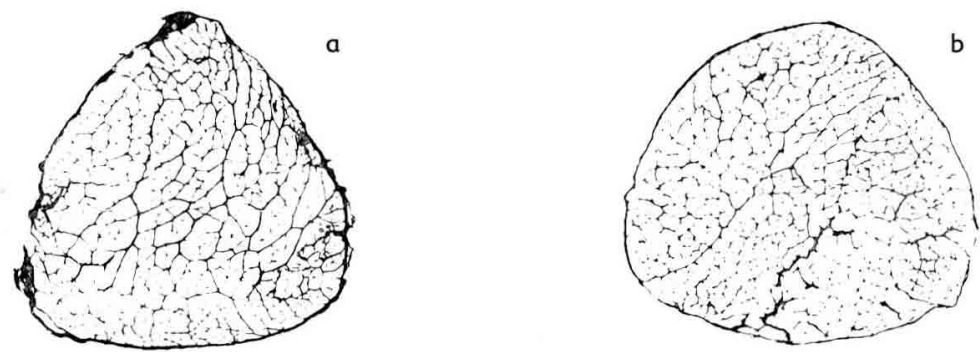

FIG. 2. - Demi-tendineux de vache de 13 ans (b)

ramené da la taille de celui du veau femelle à la naissance (a)

Chez le veau nouveau-né ce type de conjonctif est nettement mis en évidence à l'intérieur de chaque myoskhène où il dessine sous forme de lignes régulières les limites de séparation des divers faisceaux de fibres. Chez le sujet âgé (fig. 4) on distingue beaucoup plus difficilement ces séparations qui, estompées et réduites, paraissent très irrégulières d'aspect, notamment quant à leur-épaisseur.

Le tissu conjonctif qui délimite chacun des myoskhènes se présente chez le nouveau-né sous la forme d'une paroi d'épaisseur relativement constante, le long d'un côté du myoskhène. Chez le sujet âgé on assiste à un rétrécissement des parois latérales des unités qui semblent être en état de tension élastique, et qui coïncide avec un renforcement de leurs sommets. Cette image histologique conduit à penser qu'au cours de la croissance du muscle et à l'issue de son développement en épaisseur tout se passe comme si la trame initiale de tissu conjonctif structurel, présente à la naissance, se distendait à tous niveaux, sous la pression exercée par les fibres musculaires en voie d'accroissement. Ce phénomène est particulièrement perceptible au niveau de la trame qui délimite les grandes unités d'organisation de la structure musculaire, dont l'importance résulterait, peut-être, du rôle qu'elles ont à jouer dans l'organisation du muscle au cours de son développement dans le plan de croissance de l'animal.

Chez le sujet âgé l'épaississement relatif des sommets (nœuds) des unités d'organisation par rapport à leurs côtés, ce qui leur donne un aspect radié, peut provenir d'un accroissement de cette zone de la trame conjonctive où sont situées des voies de passage importantes pour les éléments intramusculaires des systèmes nerveux et circulatoire. Il peut résulter aussi (et en même temps) d'une plus grande résistance à l'extensibilité des nœuds du réseau de la trame conjonctive, ce qui protègerait ces voies de passage d'une compression préjudiciable à leur fonctionnement. Cette hypothèse implique que des différences de composition ou de structure existent entre les nouds et les autres parties du périmysium.

Ces observations effectuées dans le cas du muscle semi-tendinosus amènent donc à penser que l'accroissement de dimension des fibres au cours de la croissance de ce muscle, s'accompagne, chez les bovins, d'une dilatation différentielle de la trame conjonctive existant à la naissance. Les observations similaires que nous avons effectuées sur d'autres types de muscle permettent d'affirmer qu'il s'agit là d'un phénomène très général de la croissance musculaire des mammifères.

Reçu pour publication en mai 1972. 


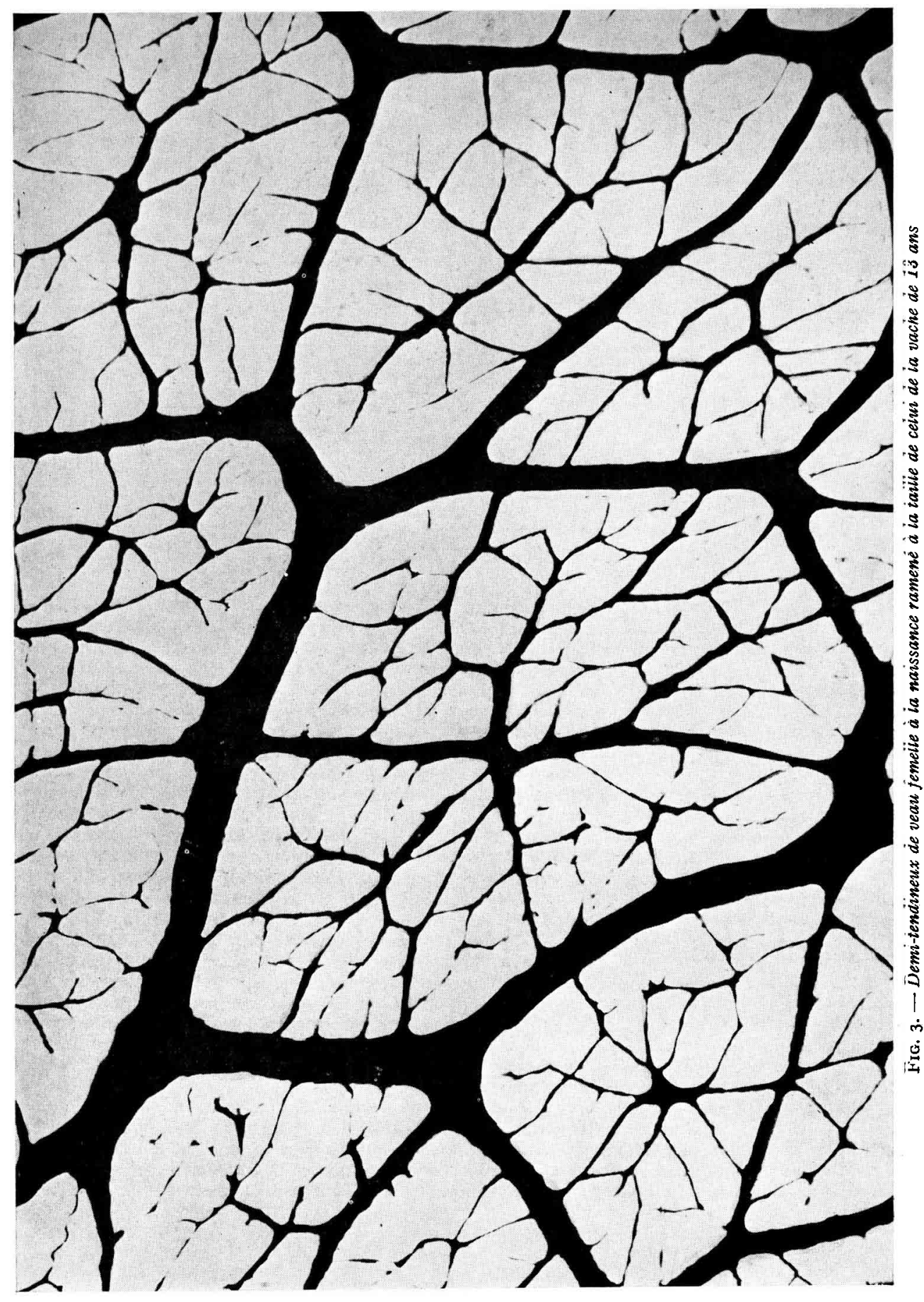




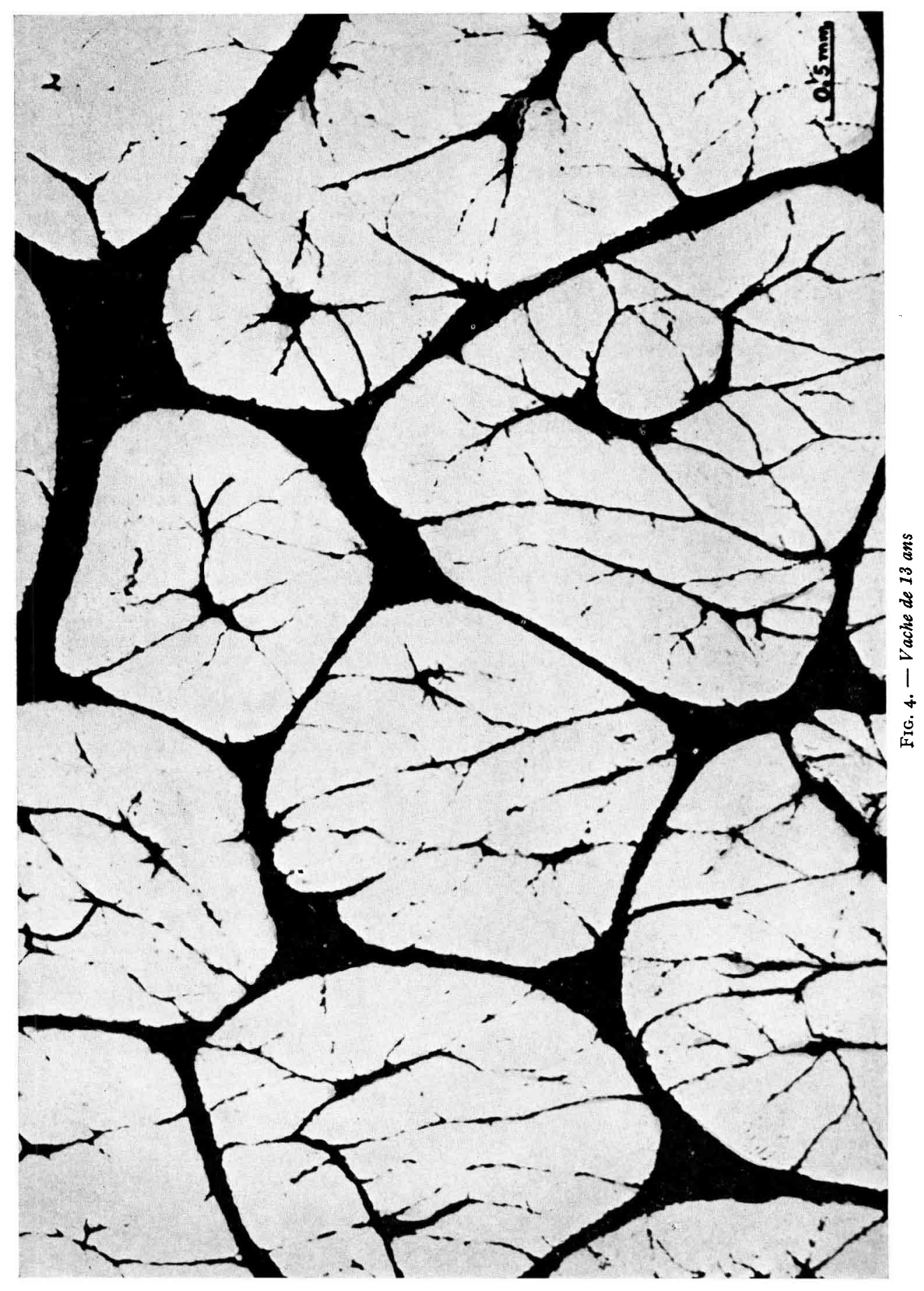




\section{SUMMARY}

\section{CONNECTIVE TISSUE FRAMEWORK AND MUSCLE GROWTH}

Histological cuts of semi-tendinosus muscle from one new born calf and one $13^{\mathrm{d}}$ years old cow were compared at different magnifications in order to study the evolution of the framework of connective tissue with age. In the very young animal connective tissue seemed to be relatively more important than in the older. During growth, muscle enlargement resulted from a general distension of the connective network affecting irregularely each muscular structure unit. Their sides appeared thin, as if under elastic tension. Their summits, corresponding to the nodes of connective tissue framework, were enlarged and looked less extensible as if they were constituted by a connective tissue different from the other parts of the perimysium.

\section{RÉFÉRENCES BIBL,IOGRAPHIQUES}

Goll D. E., Bray R. W., Hokkstra W. G., 1963. Age associated changes in muscle composition. The isolation and properties of a collagenous residue from bovine muscle.J. Food Sci.,28, 503-509.

Goll D. E., Bray R. W., Koekstra W. G., I964. Age associated changes in bovine muscle connective tissue. III. Rate of solubilization at $100^{\circ} \mathrm{C}$. $J$. Food Sci., 29, 622-628,

Kopp J., r97r. Évolution qualitative du collagène musculaire de bovin en fonction de l'âge des animaux. Conséquences sur la tendreté de la viande. Bull. Teckn, no $5, I . N . R . A$. . C. R. Z. V., Theix.

LAWRIE R. A., I96r. Studies on the muscles of meat animals. I. Differences in composition of beef Longissimus dorsi muscles determined by age and anatomical location. J. agric. Sci., 56, 249-259

Schmit O., Dumont B. L., 1969. Méthodes d'analyse de la structure musculaire. Ann. Biol. anim. Biochim. Biophys., 9, 123-134.

Vognarová I., DVorák Z., Böhm R., r968. Collagen and elastin in different cuts of veal and beef J. Food Sci., 33, 339-343.

Wilson G. D., Bray R. W., Phillips P. H., i954. The effect of age and grade on the collagen and elastin content of beef and veal, J. anim. Sci., 13, 826-83I. 